\title{
Key Role of Human ABC Transporter ABCG2 in Photodynamic Therapy and Photodynamic Diagnosis
}

\author{
Toshihisa Ishikawa, ${ }^{1,2}$ Hiroshi Nakagawa, ${ }^{2}$ Yuichiro Hagiya, ${ }^{2}$ Naosuke Nonoguchi, ${ }^{3}$ \\ Shin-ichi Miyatake, ${ }^{3}$ and Toshihiko Kuroiwa ${ }^{3}$ \\ ${ }^{1}$ Omics Science Center, RIKEN Yokohama Institute, 1-7-22 Suehiro-cho, Tsurumi-ku, Yokohama 230-0045, Japan \\ ${ }^{2}$ Graduate School of Bioscience and Biotechnology, Tokyo Institute of Technology, Yokohama 226-8501, Japan \\ ${ }^{3}$ Department of Neurosurgery, Osaka Medical College, Osaka 569-8686, Japan
}

Correspondence should be addressed to Toshihisa Ishikawa, toshi-i@gsc.riken.jp

Received 8 November 2009; Accepted 9 May 2010

Academic Editor: Ryan F. Donnelly

Copyright (C) 2010 Toshihisa Ishikawa et al. This is an open access article distributed under the Creative Commons Attribution License, which permits unrestricted use, distribution, and reproduction in any medium, provided the original work is properly cited.

\begin{abstract}
Accumulating evidence indicates that ATP-binding cassette (ABC) transporter ABCG2 plays a key role in regulating the cellular accumulation of porphyrin derivatives in cancer cells and thereby affects the efficacy of photodynamic therapy and photodynamic diagnosis. The activity of porphyrin efflux can be affected by genetic polymorphisms in the ABCG2 gene. On the other hand, Nrf2, an NF-E2-related transcription factor, has been shown to be involved in oxidative stress-mediated induction of the $A B C G 2$ gene. Since patients have demonstrated individual differences in their response to photodynamic therapy, transcriptional activation and/or genetic polymorphisms of the $A B C G 2$ gene in cancer cells may affect patients' responses to photodynamic therapy. Protein kinase inhibitors, including imatinib mesylate and gefitinib, are suggested to potentially enhance the efficacy of photodynamic therapy by blocking ABCG2-mediated porphyrin efflux from cancer cells. This review article provides an overview on the role of human $\mathrm{ABC}$ transporter ABCG2 in photodynamic therapy and photodynamic diagnosis.
\end{abstract}

\section{Introduction}

Photodynamic therapy (PDT) and photodynamic diagnosis are achieved by a photon-induced physicochemical reaction which is induced by excitation of photosensitizer exposed to light. In the 1960s Lipson and Baldes introduced a hematoporphyrin derivative $(\mathrm{HpD})$, a product derived following by treatment of hematoporphyrin with a mixture of acetic and sulfuric acids and sodium hydroxide [1]. Their development of the hematoporphyrin derivative established the basis of today's PDT and photodynamic diagnosis [26]. PDT utilizes porphyrin derivatives to generate singlet oxygen $\left({ }^{1} \mathrm{O}_{2}\right)$ and other reactive oxygen species (ROS) that are potent in killing cancer cell in vivo [7]. The modern era of PDT was founded in the 1970s with the pioneering work of Dougherty and his coworkers who purified $\mathrm{HpD}$ later called Photofrin. In 1978, Dougherty et al. had carried out the first human trials of Photofrin on women with advanced breast cancer [8]. Photofrin is still the most widely used photosensitizer in clinical PDT. Recent studies of modern PDT began just two decades ago; therefore there are still unsolved problems. Nevertheless, PDT has many applications in a wide range of fields of both preclinical and clinical sciences.

In recent years, remarkable advances were made in photodynamic diagnosis technology that makes it easier to reliably achieve complete excision of malignant gliomas [911] and meningiomas [12]. The extent of tumor resection that should be undertaken in patients with glioblastoma multiforme remained controversial [13, 14]. Fluorescenceguided gross-total resection has been developed and it has prolonged the survival time of glioblastoma and meningioma patients $[9-12,15,16]$. Historically, two fluorescent agents, that is, fluorescein sodium and protoporphyrin IX (PpIX) induced by $\delta$-Aminolevulinic acid (ALA) or its ester, have been used in glioma surgery. Because of its high tumor specificity and safety, ALA is particularly promising. It actively accumulates in the neoplasm and is converted 
to PpIX which is fluorescent. This phenomenon has been clinically applied to the detection of neoplasms in the brain and other organs, such as the bladder, skin, and bronchus. This technique is generally termed fluorescence diagnosis or photodynamic diagnosis. Fluorescence-guided resection may be beneficial for the removal of complicated or malignant tumors that have a high risk of recurrence. The most important point in fluorescence-guided microsurgery is the use of good equipment that can provide sufficient operative fields even under fluorescence mode.

\section{Biosynthesis and Metabolism of Porphyrins and Heme}

Hemes play critical roles in diverse biological processes, such as respiration and oxidative metabolism [17, 18]. Both heme biosynthesis and its intracellular concentration are tightly regulated. Heme molecules are formed via an eight-stepped pathway that is spatially shared between mitochondria and cytoplasmic compartments (Figure 1). At the first step, ALA is synthesized from glycine and succinyl Co-A in a reaction catalyzed by ALA-synthase and regulated by the intracellular-free heme pool. After ALA synthesis, a sequence of reactions occur leading to the production of various porphyrin compounds. Finally, as a result of ferrochelatase action, ferrous iron is incorporated into PpIX to form heme. Heme production as well as the synthesis of its two immediate precursors (PpIX and protoporphyrinogen) occurs in the mitochondria. ABCB6, one of human $\mathrm{ABC}$ transporters, reportedly transports coproporphyrinogen III from the cytoplasm to the mitochondria [19], whereas another ABC transporter ABCG2 is responsible for the cellular homeostasis of porphyrins and their related compounds [20]. Disturbances in cellular heme biosynthesis or metabolism are associated with several types of porphyria, which represent an elevation of toxic hemeprecursors, for example, protoporphyrins [2123].

\section{Enforced Biosynthesis of Protoporphyrin IX in Cancer Cells by ALA Administration}

Exogenous ALA administration short-circuits the first step of porphyrin biosynthesis (Figure 1), where ALA is transported into cancer and normal cells by oligopeptide transporter 1 or 2 (PEPT1 or PEPT2). ALA induces the accumulation of detectable amounts of PpIX in certain types of cells, including cancer cells, making them photosensitive [24]. ALA-induced endogenous PpIX accumulation thus constitutes a photosentization process in which the selectivity of neoplastic cells in synthesizing and/or accumulating PpIX may be exploited to enhance the efficacy of PDT and photodynamic diagnosis.

PpIX has many other advantages. It is an essentially monomeric compound with a high fluorescence yield [25] and photosensitizing capability due to its good singlet oxygen quantum efficiency $[26,27]$ and is rapidly metabolized in vivo [28]. Animal and human studies have shown that
ALA induces PpIX clearance from the skin within 24 hours after systemic, topical, or intradermal administration [24], whereas hematoporphyrin derivatives cause prolonged skin photosensitivity ( 1 to 2 months).

Not all cell lines can synthesize PpIX in vitro after ALA incubation in order to provide the reproducible in vitro assays required for in vivo studies of ALA-induced PDT. HepG2, a human hepatocarcinoma cell line, has been enzymatically well characterized to synthesize PpIX endogenously from exogenous ALA [29].

\section{Photodynamic Diagnosis and Fluorescence-Guided Microsurgery}

In photodynamic diagnosis and fluorescence-guided neurosurgery $[9,10,12,15,16]$, ALA is used for intraoperative labeling of the border regions of malignant gliomas infiltrated by alive clonogenic tumor cells and is helpful in precise resection of those regions. ALA is converted to PpIX in the body and emits red fluorescence, with the excitation of blue-violet light. As PpIX preferentially accumulates in the tumor tissue in comparison with normal tissue, this red fluorescence becomes a good hallmark for discrimination between normal and tumor tissues, especially in malignant gliomas, which have infiltrative characteristics. Approximately $80 \%$ to $90 \%$ of the malignant gliomas show this red fluorescence in surgery, while only a limited number of metastatic brain tumor cases do not. In the surgery for metastatic brain tumor and lesionectomy for radiation necrosis and neurodegenerative disease, white matter around the lesion shows weak and vague fluorescence, which also provides us with a hallmark in the surgery. Additionally, in meningioma, some tumors showed the red fluorescence, which is especially helpful in the removal of the infiltrative portion in the bone and normal parenchyma [12]. Clinical data indicate that ALA-photodynamic diagnosis-assisted resection of malignant gliomas may result in statistically significant prolongation of postoperative survival $[15,16]$. Ongoing research concentrates also on the use of ALA for a selective elimination of glioma cells in situ and on lipophilic ALA derivatives with more favorable pharmacokinetic properties.

There is a question still remaining unanswered, namely: Why does PpIX accumulate in the tumor tissue more preferentially than in normal tissue? In order to answer for this question, we analyzed the expression levels of major enzymes and transporters involved in biosynthesis and metabolism of porphyrin. To determine mRNA levels of those enzymes and transporters, we design quantitative PCR primers (Table 1) and then compared their expression profiles between glioma and normal tissues (Figure 2(a)). The mRNA level of ABC transporter ABCG2 was found to be significantly lower in malignant glioma cells in the brain tumor that exhibited strong fluorescence of PpIX after ALA treatment (Figure 2(b)), whereas the surrounding normal cells emitted weak and vague fluorescence. These results suggest that $\mathrm{ABCG} 2$, a porphyrin efflux pump, is downregulated in tumors and thereby PpIX is facilitated to accumulate in cancer cells. 


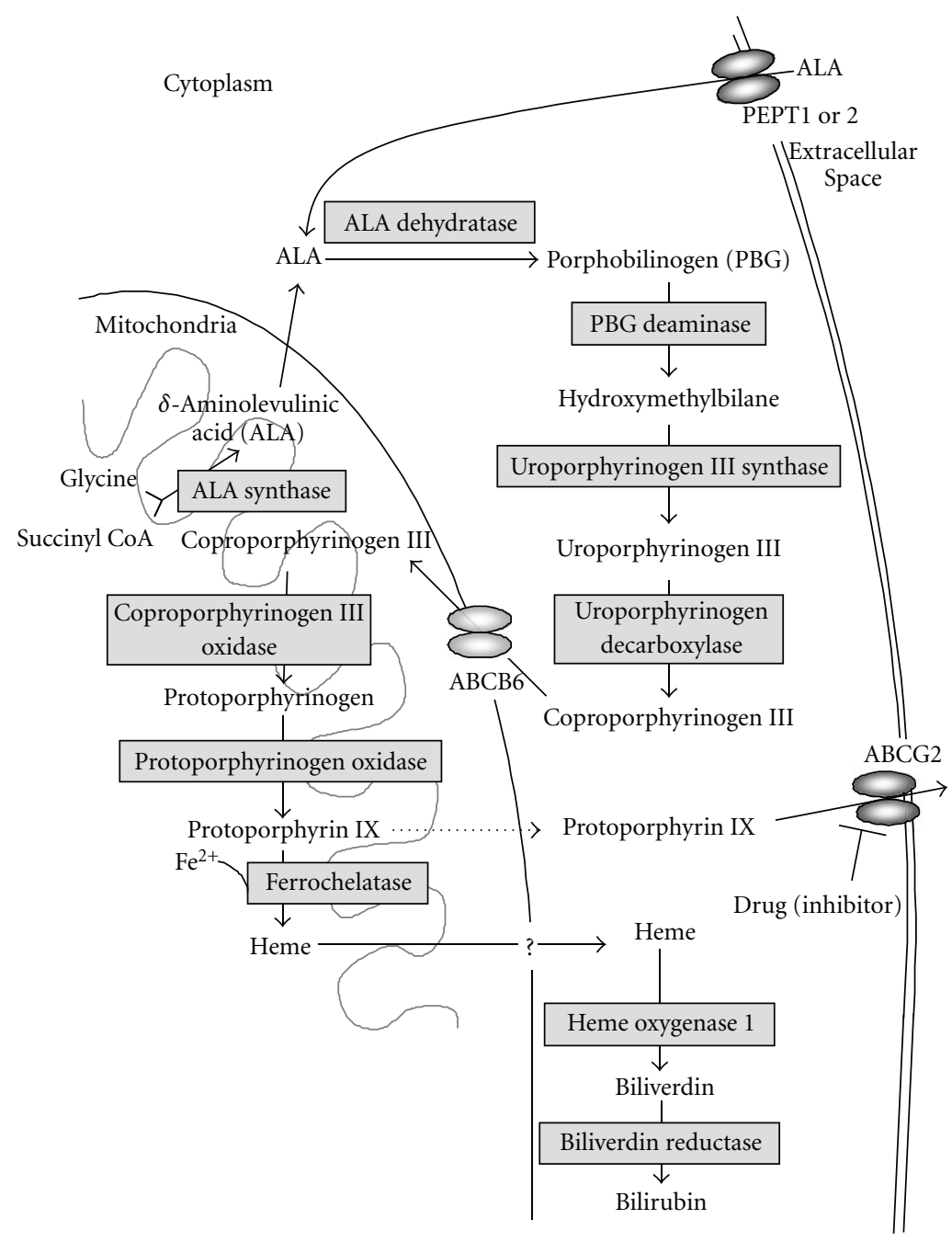

FIGURE 1: Schematic illustration of the biosynthesis and catabolism of heme. Rectangles indicate the enzymes involved in heme metabolism. Heme molecules are synthesized from glycine and succinyl Co-A via eight-stepped enzymatic reactions. ALA: $\gamma$-aminolevulinic acid; PBG: porphobilinogen. Heme, thus formed, is catabolized to biliverdin by the microsomal enzyme heme oxygenase 1. Biliverdin is subsequently metabolized to bilirubin by biliverdin reductase. ABC transporter ABCB6 is considered to be responsible for the import of coproporphyrinogen III into mitochondria, whereas ABCG2 transports porphyrins across the plasma membrane to maintain intracellular porphyrin homeostasis. ABCG2-mediated porphyrin transport can be inhibited by drugs, such as protein kinase inhibitors, as shown in Figure 5 .

\section{Human ABC Transporter ABCG2 in Cancer Chemotherapy and PDT}

Human ABC transporter ABCG2, originally named Breast Cancer Resistant Protein (BCRP), was first discovered in doxorubicin-resistant breast cancer cells [30]. Since the same transporter has also been found in the human placenta [31] as well as in drug-resistant cancer cells selected in mitoxantrone [32], the transporter was also called $\mathrm{ABCP}$ or MXR1. The ABCG2 gene is located on chromosome $4 \mathrm{q} 22$ and spans over $66 \mathrm{~kb}$, comprising 16 exons and 15 introns. ABCG2 is classified in the G-subfamily of human ABC transporter genes according to the designated international nomenclature. Compared with the molecular structures of the well-known multidrug resistance transporters $\mathrm{ABCB} 1$ (Pgp/MDR1) and ABCC1 (MRP1), ABCG2 is a so-called "half
$\mathrm{ABC}$ transporter" bearing six transmembrane domains and one ATP-binding cassette. Human ABCG2 has recently been shown to exist in the plasma membrane as a homodimer bound through disulfide-bonded cysteine residues $[16,33$, 34]. Treatment with mercaptoethanol reduced the apparent molecular weight of ABCG2 from 140,000 to 70,000. Based on the cDNA sequence, a total of eleven cysteine residues exist in the ABCG2 protein. Among them, three cysteine residues in the extra-cellular loop of ABCG2 play pivotal roles in homodimer formation or protein expression levels. While Cys603 is involved in homodimer formation, Cys592 and Cys608 appear to be even more important for the formation of an intramolecular disulfide bond that greatly affects the protein stability as well as plasma membrane targeting of the ABCG2 protein $[34,35]$. Recent studies have demonstrated that the $N$-linked glycan bound to Asn596 
TABLE 1: PCR primers to quantitatively measure the mRNA levels of PEPT1, PEPT2, ALAS1, ALAD, HMBS, UROS, UROD, ABCB6 CPOX, PPOX, ABCG2, FECH, HO-1, and HIF1alpha.

\begin{tabular}{|c|c|c|c|c|}
\hline Gene & $\mathrm{F} / \mathrm{R}$ & Primer sequence & Position & $\operatorname{Tm}$ \\
\hline PEPT1 & Forward & CCCTGAAGTGAAGGTGTTTGAAGATA & $1772-1797$ & 60.6 \\
\hline NM_005073 & Reverse & GAATTGGCCCCTGACATGAA & $2168-2188$ & 59.3 \\
\hline PEPT2 & Forward & CTACCACAATATGCCCTGGTTACA & $1894-1917$ & 58.8 \\
\hline NM_001145998 & Reverse & GCCACTGAACTGTGCCACAA & $2045-2064$ & 59.3 \\
\hline ALAS1 & Forward & GGATTCGAAACAGCCGAGTG & $1371-1390$ & 59.3 \\
\hline NM 000688 & Reverse & GAAGGTGATTGCTCCAAACTCAT & $1542-1564$ & 58.3 \\
\hline ALAD & Forward & CCTCGGTTCCAACCAACTGAT & $157-177$ & 59.8 \\
\hline NM_000031 & Reverse & GATAGGCTGTATGTCATCAGGAACA & $319-343$ & 58.2 \\
\hline HMBS & Forward & CAAGGACCAGGACATCTTGGAT & $835-856$ & 58.9 \\
\hline NM_000190 & Reverse & CCAGACTCCTCCAGTCAGGTACA & $984-1006$ & 59.2 \\
\hline UROS & Forward & TCAGCACTGCCTCTTCTATTTCC & $668-691$ & 58.7 \\
\hline NM_000375 & Reverse & CTGGGTGTGCAACTGTCTGATAC & $761-789$ & 58.3 \\
\hline UROD & Forward & CGGGAGTGTGTGGGGAA & $982-998$ & 57.2 \\
\hline NM_000374 & Reverse & AAGCAGACGTGAGTGTTTATGCA & $1178-1200$ & 58.6 \\
\hline ABCB6 & Forward & CAGAAGGGCCGTATTGAGTTTG & $2033-2054$ & 59.6 \\
\hline NM_005689 & Reverse & ATTGTCGGCGATGGTGTCA & $2308-2326$ & 59.5 \\
\hline CPOX & Forward & GGCGGAGATGTTGCCTAAGAC & $401-421$ & 59.7 \\
\hline NM_000097 & Reverse & AATGCTCACCCCAGCCTTTT & $709-728$ & 59.5 \\
\hline PPOX & Forward & CAGGAGTCCTGGGAATCGTGTA & $1251-1272$ & 59.9 \\
\hline NM_000309 & Reverse & TGCCTAGCTGACTCTAGTTTTTGC & $1509-1533$ & 58.1 \\
\hline $\mathrm{FECH}$ & Forward & GGAAATCCATTGTTCTCTAAGGC & $1252-1274$ & 57.0 \\
\hline NM_001012515 & Reverse & СТАAАТААСАСССТСТССАСАТСG & $1462-1485$ & 57.8 \\
\hline ABCG2 & Forward & CTAAGCAGGGACGAACAATCATC & $1188-1210$ & 58.8 \\
\hline NM_004827 & Reverse & TCCTGCTTGGAAGGCTCTATG & $1447-1467$ & 58.2 \\
\hline HIF-1alpha & Forward & GGCGCGAACGACAAGAA & $420-436$ & 58.0 \\
\hline NM 001530 & Reverse & CAAAACCATCCAAGGCTTTCA & $682-702$ & 58.7 \\
\hline $\mathrm{HO}-1$ & Forward & GCTCAAAAAGATTGCCCAGAA & $518-538$ & 58.1 \\
\hline NM_002133 & Reverse & TCACATGGCATAAAGCCCTACA & $926-947$ & 59.1 \\
\hline
\end{tabular}

PEPT1: oligopeptide transporter 1; PEPT2: oligopeptide transporter 2; ALAS1: delta-aminolevulinate synthase 1; ALAD, delta-aminolevulinate dehydratase; HMBS: hydroxymethylbilane synthase; UROS: uroporphyrinogen III synthase; UROD: uroporphyrinogen decarboxylase; ABCB6: ABC transporter B6; CPOX: coproporphyrinogen oxidase; PPOX: protoporphyrinogen oxidase; FECH: ferrochelatase; ABCG2: ABC transporter G2 (BCRP); HIF-1alpha: hypoxia inducible factor-1 alpha subunit; HO-1: heme oxygenase-1. F/R: forward or reverse primers; Tm: melting temperature.

is important for stabilizing nascent ABCG2 proteins by facilitating homodimers in the endoplasmic reticulum (ER) $[36,37]$.

ABCG2 is endogenously expressed in placental trophoblast cells, in the epithelium of the small intestine and liver canalicular membrane, as well as in ducts and lobules of the breast. In particular, the high levels of ABCG2 expression in trophoblast cells suggest that the pump is responsible either for transporting compounds into the fetal blood supply or for removing toxic metabolites. The apical localization in the epithelium of the small intestine and colon indicates a possible role of ABCG2 in regulating the uptake of p.o. administered drugs [38]. It has recently been reported that gefitinib (iressa) enhanced the oral availability of irinotecan in mice [39], suggestive of the potential inhibition of mouse $A b c g 2$ by gefitinib in the small intestine. On the other hand, ABCG2-knockout mice are extremely sensitive to the dietary chlorophyllbreakdown product pheophorbide a, which suggests that ABCG2 expressed in the small intestine plays a critical role in reducing the risk for developing diet-dependent phototoxicity and protoporphyria [40]. Importantly, ABCG2 has a high affinity to porphyrins, for example, hematoporphyrin and pheophorbide a [41]. ABCG2 transported protoporphyrin, hematoporphyrin, and pheophorbide a in an ATP-dependent manner [41-43].

\section{Nrf2-Mediated Induction of ABCG2 and HO-1 by Photoactivation of Porphyrins}

Recent evidence indicates that PDT can kill cancer cells directly by the efficient induction of apoptotic as well as nonapoptotic cell death pathways [7]. The identification of 


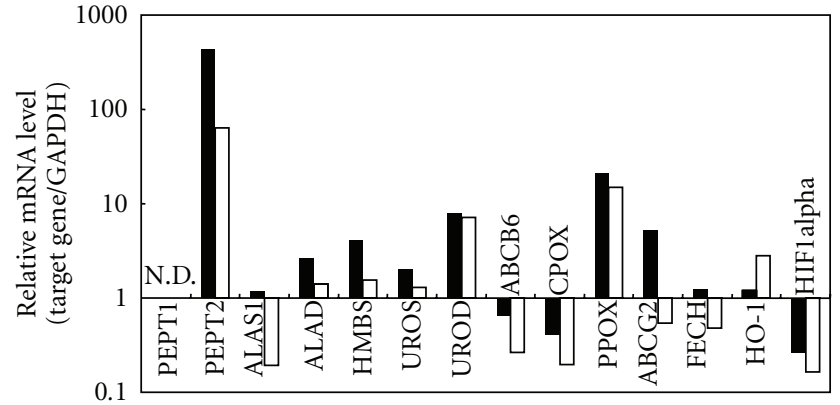

No fluorescence area

Strong fluorescence area

(a)

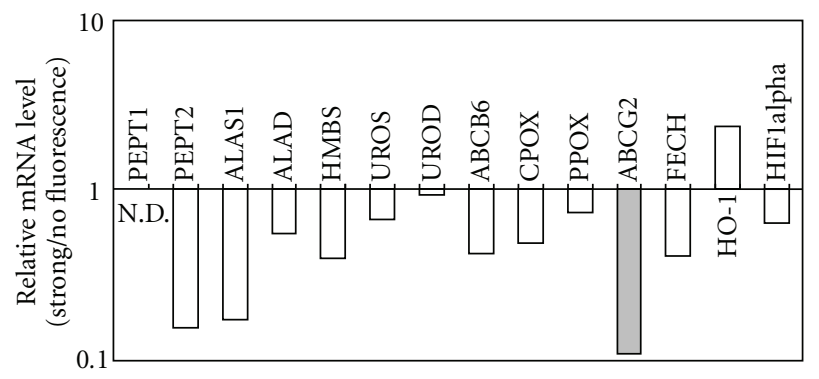

(b)

Figure 2: The mRNA levels of PEPT1, PEPT2, ALAS1, ALAD, HMBS, UROS, UROD, ABCB6 CPOX, PPOX, ABCG2, FECH, HO1 , and HIF1alpha in malignant glioma cells in the brain tumor and in the surrounding normal cells. Total RNA was extracted from strong fluorescence-emitting areas (brain tumor) as well as from the surrounding area (mostly normal tissue) without porphyrin fluorescence. The first strand cDNA was prepared from the extracted total RNA in a reverse transcriptase (RT) reaction. (a) The mRNA levels of the genes involved in the heme synthesis and metabolism reactions were detected by quantitative PCR and normalized to the mRNA level of GAPDH. (b) The relative mRNA levels of those genes in both areas were compared, where the relative mRNA level in the strong fluorescence-emitting area (brain tumor) was divided by that in nonfluorescence area (mostly normal tissue).

the molecular effectors regulating the cross-talk between cell death and cell protection pathways is an area of intense interest in the study of photokilling in cancer cells.

ABCG2 and heme oxygenase-1 (HO-1) are induced in HepG2 cells by photoactivation of porphyrins and display different induction patterns (Figures $3(\mathrm{a})$ and $3(\mathrm{~b})$ ). The induction of HO-1 was rapid and transient, whereas that of ABCG2 was relatively slow. Nevertheless, Nrf2-specific siRNA treatments caused significant impairments in the induction of both ABCG2 and HO-1 after the photoactivation of porphyrins [44], suggesting that Nrf2 is a common regulator for the transcriptional activation of $A B C G 2$ and $\mathrm{HO}-1$ genes.
Nrf2 is a basic region-leucine zipper- (bZip-) type transcription factor and plays a critical role in transcriptional upregulation of many target genes, including those for metabolizing enzymes and transporters essential for cellular defense in response to oxidative and/or electrophilic stress $[45,46]$. Nrf2 targets the ARE containing the consensus sequence of $5^{\prime}$-A/GTGACNNNGC) [45]. Keap1, on the other hand, is known to be a negative regulator of Nrf2 by retrieving it in the cytoplasm (Figure 3(c)). Oxidative stress and/or electrophilic attack lead to the dissociation of Nrf2 from Keap1 and thereby activate Nrf2 for transcriptional regulation of ARE-dependent genes. Indeed, many genes encoding detoxifying and antioxidant enzymes were found to be regulated by Nrf2 [46, 47].

We have recently reported the potential role of the Nrf2/Keap1 mechanism in the induction of human ABC transporters as well as HO-1 in HepG2 cells under oxidative stress $[44,48]$. The siRNA-mediated knockdown of Nrf2 or Keap1 revealed that the induction of ABCG2 and $\mathrm{HO}-1$ under oxidative stress involves an Nrf2-dependent mechanism. However, the time course of ABCG2 induction is different from that of HO-1. While the Nrf2/Keap1 mechanism may be involved in the oxidative stress-mediated ABCG2 induction, the induction mechanism may be indirect. Thus, we consider an alternative mechanism of socalled "trans-activation" for the induction of the ABCG2 gene [48].

The activation and nuclear translocation processes of Nrf2 in HO-1 induction seem to be more complex than previously expected. As shown in Figure 3(c), at least three distinct pathways may be involved in the activation of the Nrf2 protein leading to HO-1 induction: oxidation of critical cysteinyl residues of the Keap1 protein and concomitant inhibition of ubiquitination activity of Keap1 (Pathway i); phosphorylation of the Nrf2 protein via protein kinases, such as $\mathrm{p} 38^{\mathrm{MAPK}}$, PI3K, PKC, and PERK (Pathway ii); and direct binding of heme to Bach1 and facilitation of Nrf2/small Maf heterodimer formation (Pathway iii).

The HO-1 induction is dependent on transcription and de novo protein synthesis, and it is preceded by the nuclear accumulation of the Nrf2 transcription factor. In pathway (i), Nrf2 is repressed under quiescent conditions, where Keap1 and Cul3 constitute a unique ubiquitin E3 ligase that leads to the degradation of Nrf2. Upon exposure to oxidants/electrophiles, the enzymatic activity of this ligase complex is inhibited and the complex fails to degrade $\mathrm{Nrf2}$, resulting in the transcriptional activation of $\mathrm{Nrf} 2$ target genes [47, 49]. Cys151 of Keap1 reportedly plays an important role to facilitate Nrf2 activation in response to oxidants/electrophiles [49].

Regarding pathway (ii), Martin et al. [50] reported that HO-1 expression is regulated through the PI3K/Akt pathway and the Nrf2 transcription factor in response to the antioxidant phytochemical carnosol. Kocanova et al. [51] more recently characterized the signaling pathways and the mechanisms leading to the upregulation of HO-1 in cancer cells subjected to hypericin-based PDT. They have shown that $\mathrm{HO}-1$ induction mechanisms involve the $\mathrm{p} 38^{\mathrm{MAPK}}$ and PI3K signaling cascade. Besides $\mathrm{p} 38^{\mathrm{MAPK}}$ and PI3K, the 


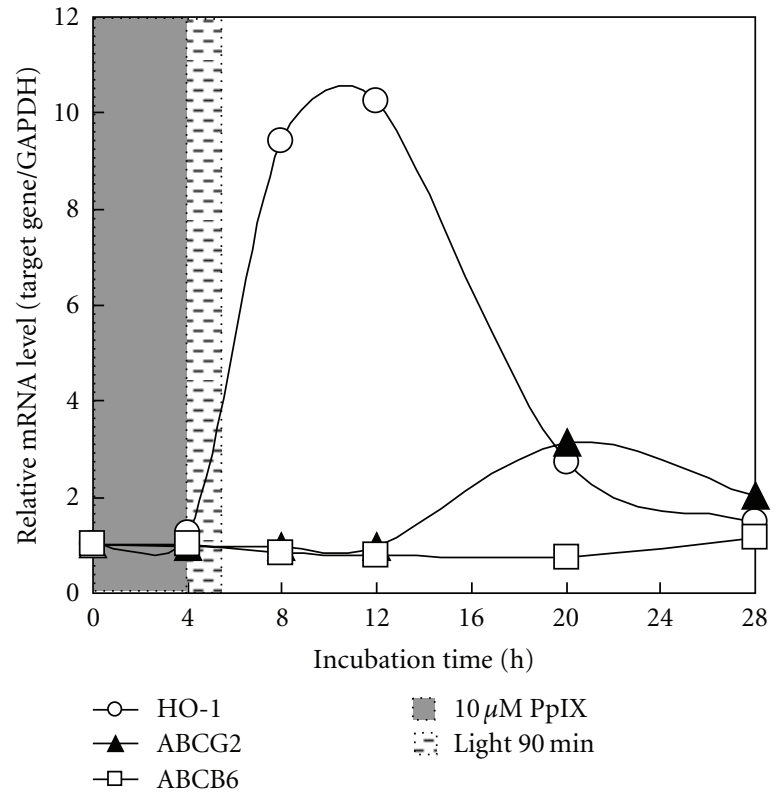

(a)

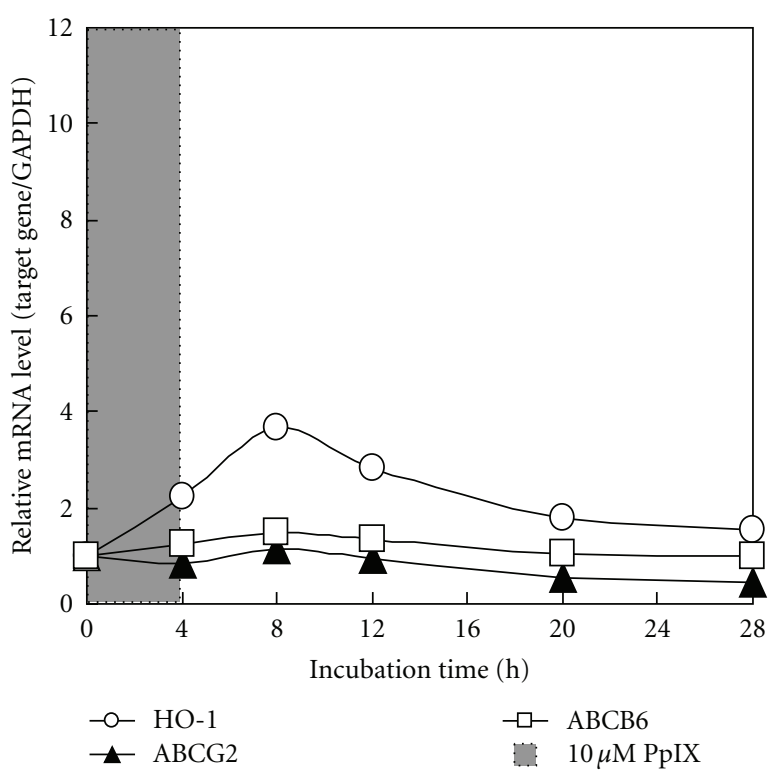

(b)

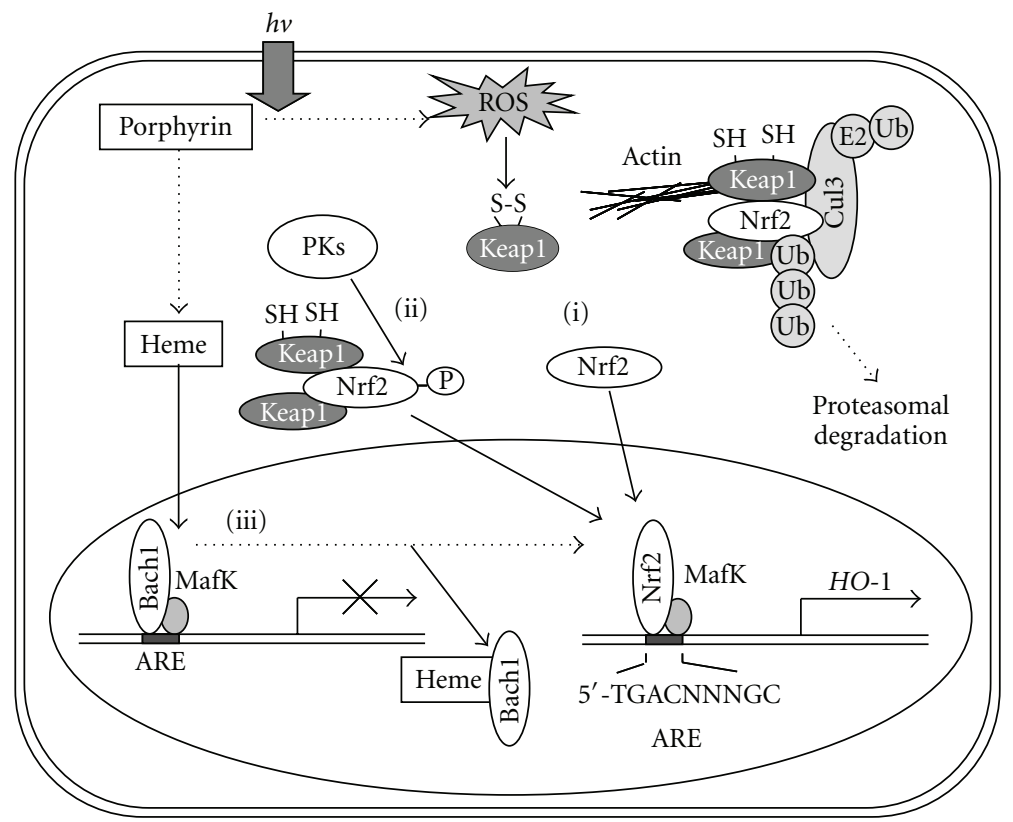

(c)

FIGURE 3: (a) Time courses of changes in the mRNA levels of heme oxygenase-1 (HO-1), ABCG2, and ABCB6 in HepG2 cells incubated with protoporphyrin IX (PpIX) and exposed to visible light. In dark conditions, HepG2 cells $\left(1 \times 10^{6}\right.$ cells $)$ were first incubated with $10 \mu \mathrm{M}$ PpIX in Dulbecco's modified Eagle's medium at $37^{\circ} \mathrm{C}$ under $5 \% \mathrm{CO}_{2}$ gas for 4 hours. Thereafter, the incubation medium was replaced with fresh incubation medium. Cells were then exposed to visible light for $90 \mathrm{~min}$, where cell culture dishes containing HepG2 cells were placed on a light viewer (Hakuba Model 5700) in an incubation chamber $\left(37^{\circ} \mathrm{C}, 5 \% \mathrm{CO}_{2}\right)$. Cells were subsequently incubated in the dark at $37^{\circ} \mathrm{C}$ under $5 \% \mathrm{CO}_{2}$ gas for 22.5 hours. The whole incubation was performed for a total of 28 hours, as indicated in the figure. (b) As the control experiments without light exposure, HepG2 cells $\left(1 \times 10^{6}\right.$ cells $)$ were incubated with $10 \mu \mathrm{M}$ PpIX in the same way as described above. PCR primers to quantitatively measure mRNA levels of HO-1, ABCG2, ABCB6, and GAPDH are described in [44]. (c) Schematic illustration for the activation of Nrf2 via three different pathways ((i), (ii), and (iii)). Pathway (i), under homeostatic conditions, Nrf2 is sequestered in the cytoplasm by the Keap1-Cul3 complex and rapidly degraded in a ubiquitin-proteasome-dependent manner. After an oxidative challenge (e.g., ${ }^{1} \mathrm{O}_{2}$ ), oxidation of two reactive cysteine residues of Keap1 inhibits the ubiquitination reaction of Nrf2 mediated by the Keap1-Cul3 complex, which results in both cytoplasmic accumulation and nuclear translocation of Nrf2. Pathway (ii), activation of Nrf2 is mediated by protein kinases (PKs), such as $\mathrm{p} 38^{\mathrm{MAPK}}$, PI3K, PERK, and PKC. Pathway (iii), under normal conditions, the chromatin structure of HO-1 is in a preactivation state, but transcription is repressed by Bach1. Heme binds to Bach1, inhibiting its DNA binding activity and inducing its nuclear export. In the nuclei, the activated Nrf2 dimerizes with small Maf nuclear protein for effective binding to the ARE consensus sequence in the promoter region of the $\mathrm{HO}-1$ gene. 
activation of Nrf2 is mediated by other protein kinases, such as PERK and PKC, being dependent on cell types [52-57].

In pathway (iii), heme regulates the dynamic exchange of Bach1 and Nrf2 in the Maf transcription factor network. Igarashi and his colleagues proposed this direct interaction model [58-61]. The transcription repressor Bach1 is a sensor and effector of heme that regulates the expression of $\mathrm{HO}$ 1 and globin genes [62-65]. Under normal conditions, the chromatin structure of $\mathrm{HO}-1$ is in a preactivation state, but transcription is repressed by Bach1. Heme binds to Bach1, inhibiting its DNA binding activity [66] and inducing its nuclear export [67]. Furthermore, heme induces ubiquitination and degradation of the transcription factor Bach1 [68]. As a consequence, heme induces the switching of $\mathrm{Nrf2} /$ small Maf hetero-dimers, resulting in HO-1 expression [69].

\section{Clinical Implications of Nrf2-Mediated Induction of HO-1}

The induction of HO-1 after photoactivation of PpIX or Pheo a is most probably mediated by pathway (i), whereby ${ }^{1} \mathrm{O}_{2}$ generated from the photooxidative reaction could oxidize the critical cysteine residues of Keap1 and thereby increase the nuclear availability of the Nrf2 pool. HO-1 catalyzes the oxidation of heme to biologically active products: carbon monoxide (CO), biliverdin, and ferrous iron. It participates in maintaining cellular homeostasis and plays an important protective role in the tissues by reducing oxidative injury, attenuating the inflammatory response, inhibiting cell apoptosis, and regulating cell proliferation. Induction of HO-1 by hemin prior to irradiation is cytoprotective [51]. A growing body of evidence indicates that $\mathrm{HO}-1$ activation plays a pivotal role in the progression of tumors. HO- 1 is very often upregulated in tumor tissues, and its expression is further increased in response to therapies. Many studies have convincingly shown that upregulation of $\mathrm{HO}-1$ significantly improves survival of hepatoma, melanoma, thyroid carcinoma, chronic myelogenous leukemia, gastric cancer, and colon cancer cell lines [70]. Increased transcription and translation of HO-1 was observed in Chinese hamster fibroblasts following photodynamic stress or photofrin II incubation [71]. Nowis et al. recently demonstrated that HO1 protected tumor cells against PDT-mediated cytotoxicity [72]. On the other hand, HO-1 inhibitors or targeted knockdown of HO-1 expression made the cultured cell lines much more sensitive to anticancer therapy. Accordingly, inhibition of $\mathrm{HO}-1$ can be suggested as a potential therapeutic approach sensitizing tumors to radiation, chemotherapy, or PDT [70].

\section{Pharmacogenomics of ABCG2 and Potential Impact on PDT}

Single nucleotide polymorphisms (SNPs) of the ABCG2 gene have been suggested to be a significant factor affecting patients' responses to medication and/or the risk of diseases [73-77]. Sequencing of the $A B C G 2$ gene from human samples has revealed over 80 different, naturally occurring sequence variations [75-77]. Among them, a total of 17 nonsynonymous polymorphisms have been reported for the ABCG2 gene $[41,74,78]$ (Figure 4(a)).

The most extensively studied among those SNPs with potential clinical relevance is $421 \mathrm{C}>\mathrm{A}$ resulting in a glutamine to lysine substitution $(\mathrm{Q} 141 \mathrm{~K})$ in the ABCG2 protein. The Q141K SNP has been identified with varying frequencies in different ethnic groups and was found to be the most relevant in Japanese and Chinese populations (approximately $30 \%$ in the allele frequency). Low allele frequencies $(0 \%$ to $35 \%)$ are found in the Africans North of the Sahara, sub-Saharan Africans, and African-American subjects [74].

Q141K has been associated with lower levels of protein expression and impaired transport in vitro [79-84]. The polymorphism has been studied in vivo; patients carrying the SNP were found to have elevated plasma levels of gefitinib, diflomotecan, and increased bioavailability of oral topotecan [85-87]. Furthermore, the Q141K SNP was reportedly associated with a higher incidence of diarrhea in nonsmall cell lung cancer patients treated with gefitinib [88]. It has been demonstrated that the reduced expression levels of the $\mathrm{Q} 141 \mathrm{~K}$ variant may be due to its ubiquitin-mediated proteasomal degradation [89].

The apparent Km value of ABCG2 WT toward hematoporphyrin was estimated to be $17.8 \mu \mathrm{M}$ [41]. To clarify the possible physiological or pathological relevance of ABCG2 polymorphisms, we have functionally validated polymorphisms of ABCG2 [19, 41, 42, 78]. Based on the currently available data on SNPs and acquired mutations, we have created a total of 18 variant forms of ABCG2 (V12M, G51C, Q126stop, Q141K, T153M, Q166E, I206L, F208S, S248P, E334stop, F431L, S441N, R482G, R482T, F489L, F571I, N590Y, and D620N) by site-directed mutagenesis and expressed them in insect cells [41, 90]. The variants Q126stop, F208S, S248P, E334stop, and S441N are defective in the transport of hematoporphyrin (Figure 4(b)). The F489L variant showed impaired transport activity (Figure 4(b)). Flp-In-293 cells expressing the F208S, S248P, S441N, and F489L variants were sensitive to light when cells were treated with pheophorbide a. Thus, it is likely that humans with these alleles may be more susceptible to porphyrin-induced phototoxicity.

\section{Interaction of ABCG2 with Protein Kinase and CDK Inhibitors}

Protein kinases are potential drug targets for the treatment of a variety of diseases, including cancer [91]. In particular, specific tyrosine kinase inhibitors are rapidly being developed as new drugs for the inhibition of malignant cell growth and metastasis. Most of these newly developed tyrosine kinase inhibitors are hydrophobic and thus rapidly penetrate the cell membrane to reach intracellular targets.

The human genome encodes more than 500 protein kinases, and this protein kinase family has been the subject of intensive research for the development of novel anticancer drugs [92]. Gefitinib and imatinib are new anticancer drugs 


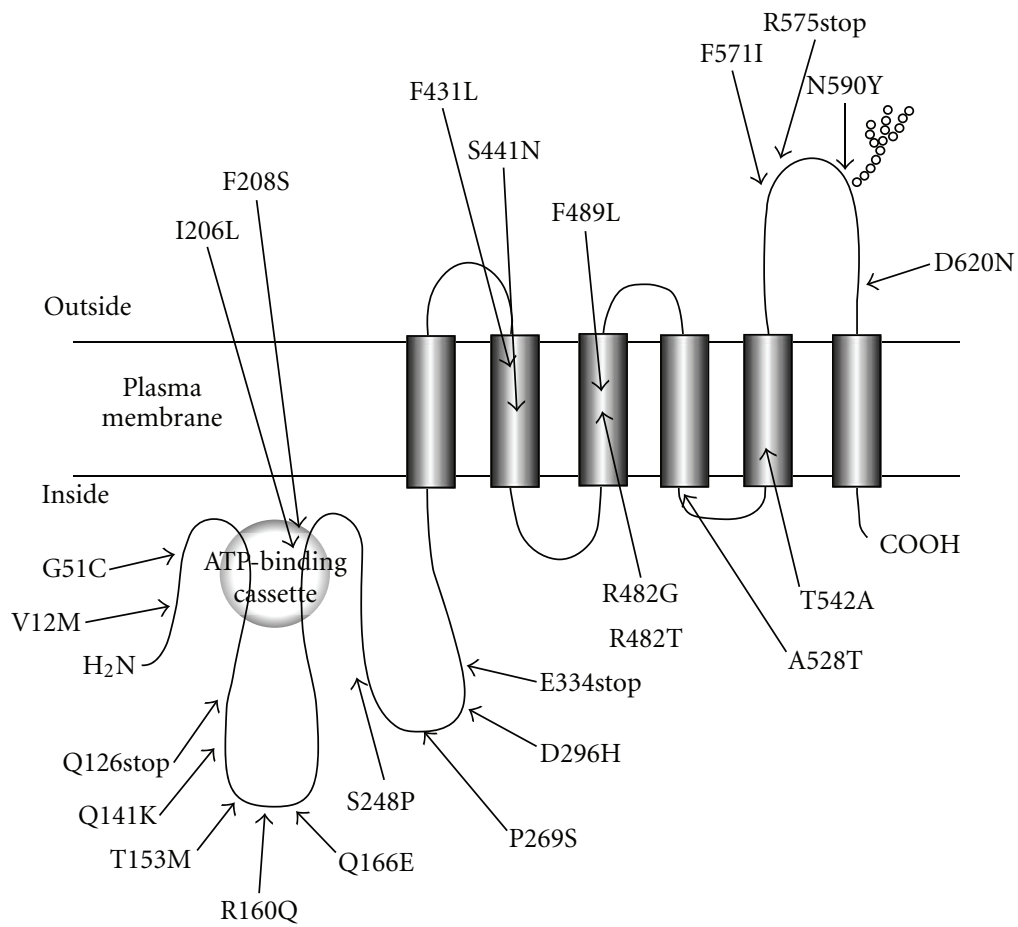

(a)

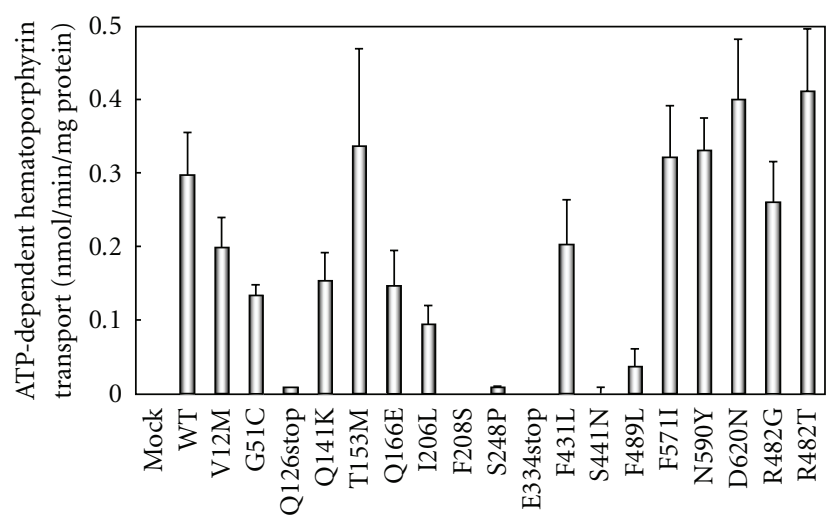

(b)

FIGURE 4: (a) Schematic illustration of human ABCG2 and its nonsynonymous polymorphisms. SNP data on the polymorphisms of ABCG2 were obtained from the NCBI dbSNP database and recent publications. The variants R482G and R482T are acquired mutations. (b) ATPdependent transport of hematoporphyrin by ABCG2 and its variants. Plasma membrane vesicles expressing ABCG2 variants (50 $\mu \mathrm{g}$ of protein) were incubated with $20 \mu \mathrm{M}$ hematoporphyrin in the presence or absence of $1 \mathrm{mM}$ ATP in the standard incubation medium at $37^{\circ} \mathrm{C}$ for 10 minutes. The ATP-dependent transport of hematoporphyrin is normalized for the amount of ABCG2 protein. Data are from [41].

that have been developed as inhibitors for EGFR tyrosine kinase and BCR/ABL kinase, respectively. Recently, another class family of protein kinases has been attracting particular attention, namely, the cyclin-dependent kinases (CDKs), which regulate critical processes of cell cycle progression and gene transcription essential for cancer cell survival [93]. In cancer, CDKs are deregulated in different ways, such as by the overexpression of cyclin E [94] and loss of p16 ${ }^{\mathrm{INK} 4 \mathrm{~A}}$, a CDK inhibitor [95]. Thus, small-molecule chemicals that specifically regulate or inhibit CDKs are of great interest in drug discovery and development for cancer chemotherapy.
The QSAR analysis revealed that a structure having one amine bonded to one carbon of a heterocyclic ring is an important component for interaction with the ABCG2 protein [96]. In addition, fused heterocyclic ring(s) and two substituents on a carbocyclic ring of the fused heterocyclic $\operatorname{ring}(\mathrm{s})$ are also important chemical moieties for the interaction with ABCG2 [96]. Interestingly, many protein kinase inhibitors carry such structural components within their molecules. Based on the QSAR analysis, we hypothesized that those CDK inhibitors would interact with the ABCG2 protein [37]. 


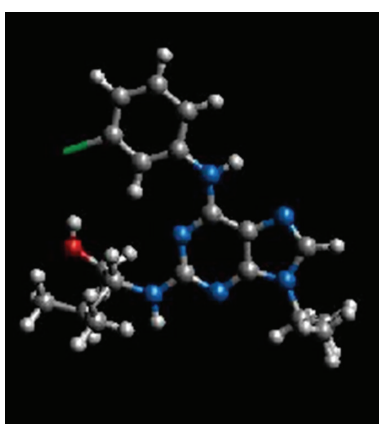

Purvalanol A

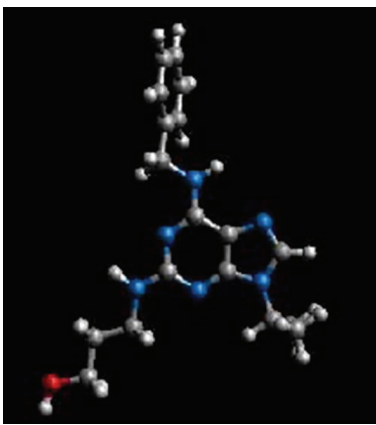

Bohemine

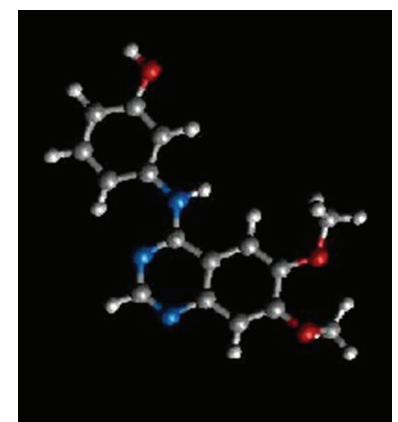

WHI-P180

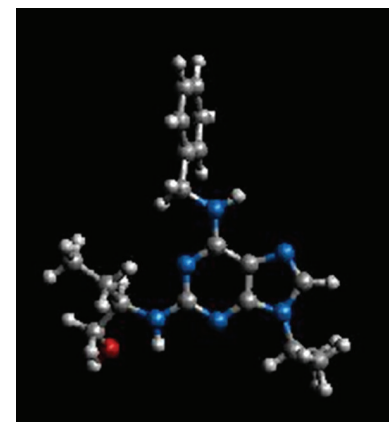

Roscotivine

(a)

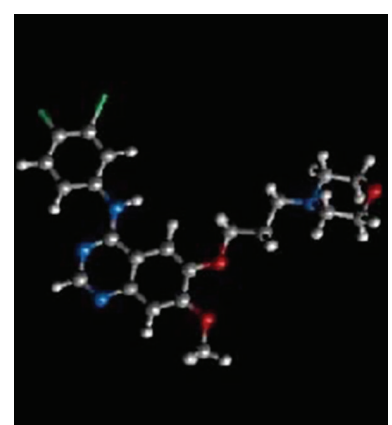

Gefitinib

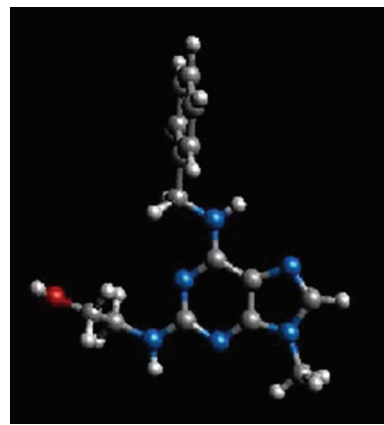

Olomoucine

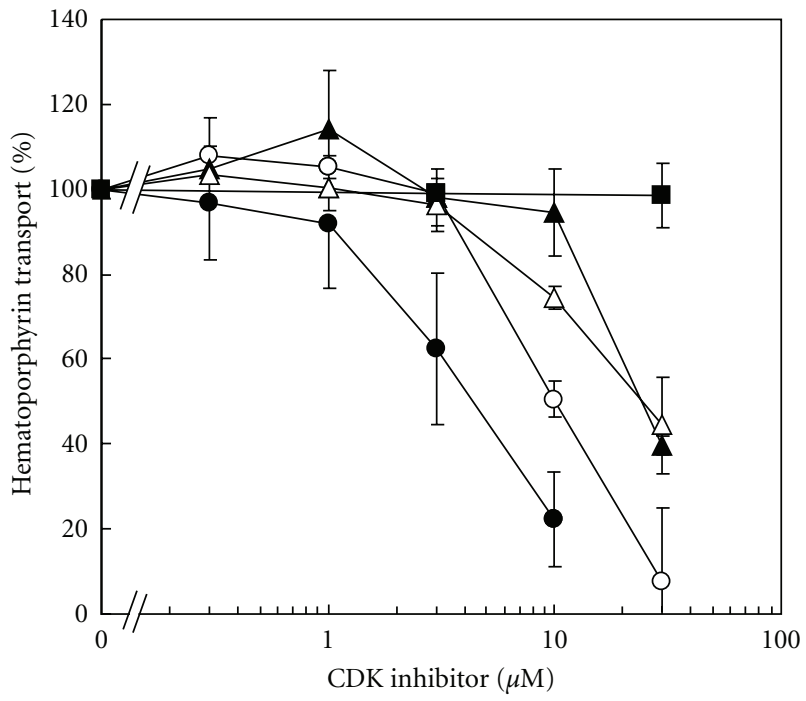

- Purvalanol A
- - WHI-P180
— Roscotivine

$\triangle$ Bohemine

$\rightarrow$ - Olomoucine

(b)

FIgUre 5: (a) Chemical structures of CDK inhibitors: purvalanol A, WHI-P180, gefitinib, bohemine, roscovitine, and olomoucine. (b) Inhibition of ABCG2-mediated hematoporphyrin transport by purvalanol A, WHI-P180, roscovitine, bohemine, or olomoucine. ABCG2expressing plasma membrane vesicles $(20 \mu \mathrm{g}$ of protein) were incubated with $20 \mu \mathrm{M}$ hematoporphyrin in the presence of purvalanol A, WHI-P180, roscovitine, bohemine, or olomoucine (final concentration: $0,0.3,1,3,10$, or $30 \mu \mathrm{M}$ ) in the standard incubation medium $\left(0.25 \mathrm{M}\right.$ sucrose and $10 \mathrm{mM}$ Tris/HEPES, pH 7.4, $1 \mathrm{mM}$ ATP, $10 \mathrm{mM}$ creatine phosphate, $100 \mu \mathrm{g} / \mathrm{mL}$ of creatine kinase, $10 \mathrm{mM} \mathrm{MgCl}_{2}$ ) at $37^{\circ} \mathrm{C}$ for $10 \mathrm{~min}$. Hematoporphyrin transported into membrane vesicles was detected [43]. Data are expressed as mean values $\pm \mathrm{SD}$ $(n=5)$. 


\section{Photosensitivity Evoked by Inhibition of ABCG2-Mediated Porphyrin Transport}

Clinical photosensitizers, such as protoporphyrin, 2-(1hexyloxethyl)-2-devinyl pyropheophorbide a (photochlor), and benzoporphyrin derivative monoacid ring $\mathrm{A}$ (Verteporfin), were transported out of cells by ABCG2, whereas this effect was abrogated by the coadministration of imatinib mesylate [97]. By increasing intracellular photosensitizer levels in ABCG2-positive tumors, imatinib mesylate or other ABCG2 transport inhibitors may enhance the efficacy and selectivity of clinical PDT [98]. In this regard, we have reported that cellular phototoxicity was evoked through the inhibition of human $\mathrm{ABC}$ transporter ABCG2 by imatinib [42] and cyclin-dependent kinase (CDK) inhibitors [43].

To gain further insights into drug-ABCG2 interactions, the three-dimensional (3D) structures of those CDK inhibitors (Figure 5(a)) were generated by $a b$ initio $\mathrm{MO}$ calculation [43]. Purvalanol A and WHI-P180 have a planar structure, whereas bohemine, roscovitine, and olomoucine do not. In the latter CDK inhibitors, the aromatic ring is orthogonal to the purine ring [43]. Among the CDK inhibitors tested (i.e., purvalanol A, WHI-P180, bohemine, roscovitine, and olomoucine), purvalanol A was found to be the most potent inhibitor for ABCG2-mediated hematoporphyrin transport (Figure 5(b)). Accordingly, it evoked the photosensitivity of ABCG2-expressing Flp-In-293 cells treated with pheophorbide a [43]. Thus, it is suggested that the planar structure is an important factor for interactions with the active site of ABCG2. Taken together, ABCG2 is one of the critical factors that affect the efficacy of PDT as well as chemotherapy of human cancer.

\section{Concluding Remarks}

ABCG2 was discovered a decade ago and has been studied in laboratories around the world, yielding a wealth of knowledge akin to that gathered for ABCB1 (Pglycoprotein/MDR1) and ABCC1 (MRP1). Chemotherapy and PDT are effective treatment options for human cancer. However, individual differences among patients were also noticed in their responses to those therapies. Gupta et al. demonstrated interesting data that ABCG2 mRNA was present in normal colorectal tissue but showed a 6-fold decrease in colorectal cancer [98]. The downregulation of ABCG2 mRNA and protein was also evident in cervical cancer. Indeed, we have also found that ABCG2 expression was downregulated in malignant glioma of human brain tumor (Figure 2). These observations indicate that cancerassociated downregulation of ABCG2 is likely to be a common phenomenon in several tumors and that the accumulation of clinical photosensitizers in cancerous tissues may be due, in part, to the reduced expression levels of ABCG2 in cancer cells. On the other hand, induction of ABCG2 and HO-1 is considered as critical factors for PDT, and they can be readily induced by photo-oxidative stress to protect cancer cells. Thus, investigations of molecular mechanisms underlying the induction of those genes would be of importance to create new strategies for individualized PDT.

\section{Acknowledgments}

The study in the authors' laboratory was supported by the Japan Science and Technology Agency (JST) Research Project, Grant-in-Aid for Scientific Research (A) (no. 18201041), and the Grant-in-Aid for Exploratory Research (no. 19659136) from the Japanese Society for the Promotion of Science (JSPS) as well as the Grant-in-Aid for Young Scientists (B) (no. 19791361) from the Ministry of Education, Culture, Sports, Science and Technology. Y. Hagiya is a JSPS Research Fellow.

\section{References}

[1] R. L. Lipson and E. J. Baldes, "The photodynamic properties of a particular hematoporphyrin derivative," Archives of Dermatology, vol. 82, pp. 508-516, 1960.

[2] R. L. Lipson, E. J. Baldes, and A. M. Olsen, "Hematoporphyrin derivative: a new aid for endoscopic detection of malignant disease," The Journal of Thoracic and Cardiovascular Surgery, vol. 42, pp. 623-629, 1961.

[3] R. L. Lipson, E. J. Baldes, and A. M. Olsen, "Further evaluation of the use of hematoporphyrin derivative as a new aid for the endoscopic detection of malignant disease," Diseases of the Chest, vol. 46, pp. 676-679, 1964.

[4] R. L. Lipson, E. J. Baldes, and M. J. Gray, "Hematoporphyrin derivative for detection and management of cancer," Cancer, vol. 20, no. 12, pp. 2255-2257, 1967.

[5] M. J. Gray, R. Lipson, J. V. Maeck, L. Parker, and D. Romeyn, "Use of hematoporphyrin derivative in detection and management of cervical cancer. A preliminary report," American Journal of Obstetrics and Gynecology, vol. 99, no. 6, pp. 766-771, 1967.

[6] D. R. Sanderson, R. S. Fontana, R. L. Lipson, and E. J. Baldes, "Hematoporphyrin as a diagnostic tool. A preliminary report of new techniques," Cancer, vol. 30, no. 5, pp. 1368-1372, 1972.

[7] D. E. J. G. J. Dolmans, D. Fukumura, and R. K. Jain, "Photodynamic therapy for cancer," Nature Reviews Cancer, vol. 3, no. 5, pp. 380-387, 2003.

[8] T. J. Dougherty, J. E. Kaufman, A. Goldfarb, KR Weishaupt, D. Boyle, and A. Mittleman, "Photoradiation therapy for the treatment of malignant tumors," Cancer Research, vol. 38, no. 8, pp. 2628-2635, 1978.

[9] W. Stummer, A. Novotny, H. Stepp, C. Goetz, K. Bise, and H. J. Reulen, "Fluorescence-guided resection of glioblastoma multiforme by using 5 -aminolevulinic acid-induced porphyrins: a prospective study in 52 consecutive patients," Journal of Neurosurgery, vol. 93, no. 6, pp. 1003-1013, 2000.

[10] F. W. Floeth and W. Stummer, "The value of metabolic imaging in diagnosis and resection of cerebral gliomas," Naure Cliical Practice Neurology, vol. 1, no. 2, pp. 62-63, 2005.

[11] J. Shinoda, H. Yano, S.-I. Yoshimura et al., "Fluorescenceguided resection of glioblastoma multiforme by using highdose fluorescein sodium. Technical note," Journal of Neurosurgery, vol. 99, no. 3, pp. 597-603, 2003. 
[12] Y. Kajimoto, T. Kuroiwa, S.-I. Miyatake et al., "Use of 5 -aminolevulinic acid in fluorescence-guided resection of meningioma with high risk of recurrence. Case report," Journal of Neurosurgery, vol. 106, no. 6, pp. 1070-1074, 2007.

[13] M. Lacroix, D. Abi-Said, D. R. Fourney et al., "A multivariate analysis of 416 patients with glioblastoma multiforme:prognosis, extent of resection, and survival," Journal of Neurosurgery, vol. 95, no. 2, pp. 190-198, 2001.

[14] S. J. Hentschel and R. Sawaya, "Optimizing outcomes with maximal surgical resection of malignant gliomas," Cancer Control, vol. 10, no. 2, pp. 109-114, 2003.

[15] W. Stummer, U. Pichlmeier, T. Meinel, O. D. Wiestler, F. Zanella, and H.-J. Reulen, "Fluorescence-guided surgery with 5-aminolevulinic acid for resection of malignant glioma: a randomised controlled multicentre phase III trial," The Lancet Oncology, vol. 7, no. 5, pp. 392-401, 2006.

[16] W. Stummer, H. J. Reulen, A. Novotny, H. Stepp, and J. C. Tonn, "Fluorescence-guided resections of malignant gliomas-an overview," Acta Neurochirurgica. Supplement, vol. 88, pp. 9-12, 2003.

[17] G. O. Latunde-Dada, R. J. Simpson, and A. T. McKie, "Recent advances in mammalian haem transport," Trends in Biochemical Sciences, vol. 31, no. 3, pp. 182-188, 2006.

[18] A. S. Tsiftsoglou, A. I. Tsamadou, and L. C. Papadopoulou, "Heme as key regulator of major mammalian cellular functions: molecular, cellular, and pharmacological aspects," Pharmacology and Therapeutics, vol. 111, no. 2, pp. 327-345, 2006.

[19] P. Krishnamurthy, T. Xie, and J. D. Schuetz, "The role of transporters in cellular heme and porphyrin homeostasis," Pharmacology and Therapeutics, vol. 114, no. 3, pp. 345-358, 2007.

[20] K. Wakabayashi, A. Tamura, H. Saito, Y. Onishi, and T. Ishikawa, "Human ABC transporter ABCG2 in xenobiotic protection and redox biology," Drug Metabolism Reviews, vol. 38, no. 3, pp. 371-391, 2006.

[21] R. Dubakiene and M. Kupriene, "Scientific problems of photosensitivity," Medicina, vol. 42, no. 8, pp. 619-624, 2006.

[22] J. T. Hindmarsh, "The porphyrias, appropriate test selection," Clinica Chimica Acta, vol. 333, no. 2, pp. 203-207, 2003.

[23] R. A. Norman, "Past and future: porphyria and porphyrins," Skinmed, vol. 4, no. 5, pp. 287-292, 2005.

[24] J. C. Kennedy and R. H. Pottier, "Endogenous protoporphyrin IX, a clinically useful photosensitizer for photodynamic therapy," Journal of Photochemistry and Photobiology B, vol. 14, no. 4, pp. 275-292, 1992.

[25] H. Schneckenburger, K. König, K. Kunzi-Rapp, C. WestphalFrösch, and A. Rück, "Time-resolved in-vivo fluorescence of photosensitizing porphyrins," Journal of Photochemistry and Photobiology B, vol. 21, no. 2-3, pp. 143-147, 1993.

[26] K. R. Weishaupt, C. J. Gomer, and T. J. Dougherty, "Identification of singlet oxygen as the cytotoxic agent in photo inactivation of a murine tumor," Cancer Research, vol. 36, no. 7, pp. 2326-2329, 1976.

[27] R. Pottier and T. G. Truscott, "The photochemistry of haematoporphyrin and related systems," International Journal of Radiation Biology, vol. 50, no. 3, pp. 421-452, 1986.

[28] C. S. Loh, D. Vernon, A. J. MacRobert, J. Bedwell, S. G. Bown, and S. B. Brown, "Endogenous porphyrin distribution induced by 5-aminolaevulinic acid in the tissue layers of the gastrointestinal tract," Journal of Photochemistry and Photobiology B, vol. 20, no. 1, pp. 47-54, 1993.
[29] F. Iwasa, S. Sassa, and A. Kappas, " $\delta$-Aminolaevulinate synthase in human HepG2 hepatoma cells. Repression by haemin and induction by chemicals," Biochemical Journal, vol. 262, no. 3, pp. 807-813, 1989.

[30] L. A. Doyle, W. Yang, L. V. Abruzzo et al., "A multidrug resistance transporter from human MCF-7 breast cancer cells," Proceedings of the National Academy of Sciences of the United States of America, vol. 95, no. 26, pp. 15665-15670, 1998.

[31] R. Allikmets, L. M. Schriml, A. Hutchinson, V. RomanoSpica, and M. Dean, "A human placenta-specific ATP-binding cassette gene (ABCP) on chromosome 4q22 that is involved in multidrug resistance," Cancer Research, vol. 58, no. 23, pp. 5337-5339, 1998.

[32] K. Miyake, L. Mickley, T. Litman et al., "Molecular cloning of cDNAs which are highly overexpressed in mitoxantroneresistant cells: demonstration of homology to ABC transport genes," Cancer Research, vol. 59, no. 1, pp. 8-13, 1999.

[33] K. Wakabayashi, H. Nakagawa, T. Adachi et al., "Identification of cysteine residues critically involved in homodimer formation and protein expression of human ATP-binding cassette transporter ABCG2: a new approach using the Flp recombinase system," Journal of Experimental Therapeutics and Oncology, vol. 5, no. 3, pp. 205-222, 2006.

[34] K. Wakabayashi, H. Nakagawa, A. Tamura et al., "Intramolecular disulfide bond is a critical check point determining degradative fates of ATP-binding cassette (ABC) transporter ABCG2 protein," Journal of Biological Chemistry, vol. 282, no. 38, pp. 27841-27846, 2007.

[35] K. Wakabayashi-Nakao, A. Tamura, T. Furukawa, H. Nakagawa, and T. Ishikawa, "Quality control of human ABCG2 protein in the endoplasmic reticulum: ubiquitination and proteasomal degradation," Advanced Drug Delivery Reviews, vol. 61, no. 1, pp. 66-72, 2009.

[36] H. Nakagawa, K. Wakabayashi-Nakao, A. Tamura, Y. Toyoda, S. Koshiba, and T. Ishikawa, "Disruption of $N$-linked glycosylation enhances ubiquitin-mediated proteasomal degradation of the human ATP-binding cassette transporter ABCG2," FEBS Journal, vol. 276, no. 24, pp. 7237-7252, 2009.

[37] T. Ishikawa and H. Nakagawa, "Human ABC transporter ABCG2 in cancer chemotherapy and pharmacogenomics," Journal of Experimental Therapeutics and Oncology, vol. 8, no. 1, pp. 5-24, 2009.

[38] J. D. Allen and A. H. Schinkel, "Multidrug resistance and pharmacological protection mediated by the breast cancer resistance protein (BCRP/ABCG2)," Molecular Cancer Therapeutics, vol. 1, no. 6, pp. 427-434, 2002.

[39] C. F. Stewart, M. Leggas, J. D. Schuetz et al., "Gefitinib enhances the antitumor activity and oral bioavailability of irinotecan in mice," Cancer Research, vol. 64, no. 20, pp. 74917499, 2004.

[40] J. W. Jonker, M. Buitelaar, E. Wagenaar et al., "The breast cancer resistance protein protects against a major chlorophyllderived dietary phototoxin and protoporphyria," Proceedings of the National Academy of Sciences of the United States of America, vol. 99, no. 24, pp. 15649-15654, 2002.

[41] A. Tamura, M. Watanabe, H. Saito et al., "Functional validation of the genetic polymorphisms of human ATPbinding cassette (ABC) transporter ABCG2: identification of alleles that are defective in porphyrin transport," Molecular Pharmacology, vol. 70, no. 1, pp. 287-296, 2006.

[42] A. Tamura, Y. Onishi, R. An et al., "In vitro evaluation of photosensitivity risk related to genetic polymorphisms of 
human ABC transporter ABCG2 and inhibition by drugs," Drug Metabolism and Pharmacokinetics, vol. 22, no. 6, pp. 428440, 2007.

[43] R. An, Y. Hagiya, A. Tamura et al., "Cellular phototoxicity evoked through the inhibition of human $\mathrm{ABC}$ transporter ABCG2 by cyclin-dependent kinase inhibitors in vitro," Pharmaceutical Research, vol. 26, no. 2, pp. 449-458, 2009.

[44] Y. Hagiya, T. Adachi, S.-I. Ogura et al., "Nrf2-dependent induction of human $\mathrm{ABC}$ transporter $\mathrm{ABCG} 2$ and heme oxygenase-1 in HepG2 cells by photoactivation of porphyrins: biochemical implications for cancer cell response to photodynamic therapy," Journal of Experimental Therapeutics and Oncology, vol. 7, no. 2, pp. 153-167, 2008.

[45] T. Nguyen, P. J. Sherratt, P. Nioi, C. S. Yang, and C. B. Pickett, "Nrf2 controls constitutive and inducible expression of ARE-driven genes through a dynamic pathway involving nucleocytoplasmic shuttling by Keap1," Journal of Biological Chemistry, vol. 280, no. 37, pp. 32485-32492, 2005.

[46] H. Motohashi and M. Yamamoto, "Nrf2-Keap1 defines a physiologically important stress response mechanism," Trends in Molecular Medicine, vol. 10, no. 11, pp. 549-557, 2004.

[47] M. Kobayashi and M. Yamamoto, "Nrf2-Keap1 regulation of cellular defense mechanisms against electrophiles and reactive oxygen species," Advances in Enzyme Regulation, vol. 46, no. 1, pp. 113-140, 2006.

[48] T. Adachi, H. Nakagawa, I. Chung et al., "Nrf2-dependent and -independent induction of ABC transporters ABCC1, ABCC2, and ABCG2 in HepG2 cells under oxidative stress," Journal of Experimental Therapeutics and Oncology, vol. 6, no. 4, pp. 335348, 2007.

[49] T. Yamamoto, T. Suzuki, A. Kobayashi et al., "Physiological significance of reactive cysteine residues of Keapl in determining Nrf2 activity," Molecular and Cellular Biology, vol. 28, no. 8, pp. 2758-2770, 2008.

[50] D. Martin, A. I. Rojo, M. Salinas et al., "Regulation of heme oxygenase-1 expression through the phosphatidylinositol 3kinase/akt pathway and the Nrf2 transcription factor in response to the antioxidant phytochemical carnosol," Journal of Biological Chemistry, vol. 279, no. 10, pp. 8919-8929, 2004.

[51] S. Kocanova, E. Buytaert, J.-Y. Matroule et al., "Induction of heme-oxygenase 1 requires the p38MAPK and PI3K pathways and suppresses apoptotic cell death following hypericinmediated photodynamic therapy," Apoptosis, vol. 12, no. 4, pp. 731-741, 2007.

[52] C. K. Andreadi, L. M. Howells, P. A. Atherfold, and M. M. Manson, "Involvement of Nrf2, p38, B-Raf, and nuclear factor- $\kappa \mathrm{B}$, but not phosphatidylinositol 3-kinase, in induction of hemeoxygenase-1 by dietary polyphenols," Molecular Pharmacology, vol. 69, no. 3, pp. 1033-1040, 2006.

[53] D. A. Bloom and A. K. Jaiswal, "Phosphorylation of Nrf2 at Ser40 by protein kinase $\mathrm{C}$ in response to antioxidants leads to the release of $\mathrm{Nrf} 2$ from INrf2, but is not required for Nrf2 stabilization/accumulation in the nucleus and transcriptional activation of antioxidant response element-mediated $\mathrm{NAD}(\mathrm{P}) \mathrm{H}$ :quinone oxidoreductase-1 gene expression," Journal of Biological Chemistry, vol. 278, no. 45, pp. 44675-44682, 2003.

[54] S. B. Cullinan, D. Zhang, M. Hannink, E. Arvisais, R. J. Kaufman, and J. A. Diehl, "Nrf2 is a direct PERK substrate and effector of PERK-dependent cell survival," Molecular and Cellular Biology, vol. 23, no. 20, pp. 7198-7209, 2003.

[55] S. B. Cullinan and J. A. Diehl, "PERK-dependent activation of Nrf2 contributes to redox homeostasis and cell survival following endoplasmic reticulum stress," Journal of Biological Chemistry, vol. 279, no. 19, pp. 20108-20117, 2004.

[56] K. A. Kang, K. H. Lee, J. W. Park et al., "Triphlorethol-A induces heme oxygenase-1 via activation of ERK and NF-E2 related factor 2 transcription factor," FEBS Letters, vol. 581, no. 10, pp. 2000-2008, 2007.

[57] K. W. Kang, S. J. Lee, J. W. Park, and S. G. Kim, "Phosphatidylinositol 3-kinase regulates nuclear translocation of NF-E2related factor 2 through actin rearrangement in response to oxidative stress," Molecular Pharmacology, vol. 62, no. 5, pp. 1001-1010, 2002.

[58] K. Igarashi and J. Sun, "The heme-Bach1 pathway in the regulation of oxidative stress response and erythroid differentiation," Antioxidants and Redox Signaling, vol. 8, no. 1-2, pp. 107-118, 2006.

[59] T. Oyake, K. Itoh, H. Motohashi et al., "Bach proteins belong to a novel family of BTB-basic leucine zipper transcription factors that interact with MafK and regulate transcription through the NF-E2 site," Molecular and Cellular Biology, vol. 16, no. 11, pp. 6083-6095, 1996.

[60] J. Sun, H. Hoshino, K. Takaku et al., "Hemoprotein Bach1 regulates enhancer availability of heme oxygenase-1 gene," The EMBO Journal, vol. 21, no. 19, pp. 5216-5224, 2002.

[61] J. Sun, M. Brand, Y. Zenke, S. Tashiro, M. Groudine, and $\mathrm{K}$. Igarashi, "Heme regulates the dynamic exchange of Bach1 and NF-E2-related factors in the Maf transcription factor network," Proceedings of the National Academy of Sciences of the United States of America, vol. 101, no. 6, pp. 1461-1466, 2004.

[62] K. J. Hintze, Y. Katoh, K. Igarashi, and E. C. Theil, "Bach1 repression of ferritin and thioredoxin reductasel is hemesensitive in cells and in vitro and coordinates expression with heme oxygenase1, $\beta$-globin, and $\operatorname{NADP}(\mathrm{H})$ quinone (Oxido) reductase1," Journal of Biological Chemistry, vol. 282, no. 47, pp. 34365-34371, 2007.

[63] T. Kitamuro, K. Takahashi, K. Ogawa et al., "Bach1 functions as a hypoxia-inducible repressor for the heme oxygenase-1 gene in human cells," Journal of Biological Chemistry, vol. 278, no. 11, pp. 9125-9133, 2003.

[64] Y. Shan, R. W. Lambrecht, T. Ghaziani, S. E. Donohue, and H. L. Bonkovsky, "Role of Bach-1 in regulation of heme oxygenase-1 in human liver cells: insights from studies with small interfering RNAs," Journal of Biological Chemistry, vol. 279, no. 50, pp. 51769-51774, 2004.

[65] Y. Shan, R. W. Lambrecht, S. E. Donohue, and H. L. Bonkovsky, "Role of Bach1 and Nrf2 in up-regulation of the heme oxygenase-1 gene by cobalt protoporphyrin," The FASEB Journal, vol. 20, no. 14, pp. 2651-2653, 2006.

[66] K. Ogawa, J. Sun, S. Taketani et al., "Heme mediates derepression of Maf recognition element through direct binding to transcription repressor Bach1," The EMBO Journal, vol. 20, no. 11, pp. 2835-2843, 2001.

[67] H. Suzuki, S. Tashiro, S. Hira et al., "Heme regulates gene expression by triggering Crm1-dependent nuclear export of Bach1," The EMBO Journal, vol. 23, no. 13, pp. 2544-2553, 2004.

[68] Y. Zenke-Kawasaki, Y. Dohi, Y. Katoh et al., "Heme induces ubiquitination and degradation of the transcription factor Bach1," Molecular and Cellular Biology, vol. 27, no. 19, pp. 6962-6971, 2007.

[69] J. F. Reichard, G. T. Motz, and A. Puga, "Heme oxygenase-1 induction by NRF2 requires inactivation of the transcriptional repressor BACH1," Nucleic Acids Research, vol. 35, no. 21, pp. 7074-7086, 2007. 
[70] A. Jozkowicz, H. Was, and J. Dulak, "Heme oxygenase-1 in tumors: is it a false friend?" Antioxidants and Redox Signaling, vol. 9, no. 12, pp. 2099-2117, 2007.

[71] C. J. Gomer, M. Luna, A. Ferrario, and N. Rucker, "Increased transcription and translation of heme oxygenase in Chinese hamster fibroblasts following photodynamic stress or Photofrin II incubation," Photochemistry and Photobiology, vol. 53, no. 2, pp. 275-279, 1991.

[72] D. Nowis, M. Legat, T. Grzela et al., "Heme oxygenase-1 protects tumor cells against photodynamic therapy-mediated cytotoxicity," Oncogene, vol. 25, no. 24, pp. 3365-3374, 2006.

[73] F. A. de Jong, M. J. A. de Jonge, J. Verweij, and R. H. J. Mathijssen, "Role of pharmacogenetics in irinotecan therapy," Cancer Letters, vol. 234, no. 1, pp. 90-106, 2006.

[74] T. Ishikawa, A. Tamura, H. Saito, K. Wakabayashi, and H. Nakagawa, "Pharmacogenomics of the human ABC transporter ABCG2: from functional evaluation to drug molecular design," Naturwissenschaften, vol. 92, no. 10, pp. 451-463, 2005.

[75] G. Bäckström, J. Taipalensuu, H. Melhus et al., "Genetic variation in the ATP-binding cassette transporter gene ABCG2 (BCRP) in a Swedish population," European Journal of Pharmaceutical Sciences, vol. 18, no. 5, pp. 359-364, 2003.

[76] T. M. Bosch, L. M. Kjellberg, A. Bouwers et al., "Detection of single nucleotide polymorphisms in the ABCG2 gene in a Dutch population," American Journal of PharmacoGenomics, vol. 5, no. 2, pp. 123-131, 2005.

[77] F. A. de Jong, S. Marsh, R. H. J. Mathijssen et al., "ABCG2 pharmacogenetics: ethnic differences in allele frequency and assessment of influence on irinotecan disposition," Clinical Cancer Research, vol. 10, no. 17, pp. 5889-5894, 2004.

[78] A. Tamura, K. Wakabayashi, Y. Onishi et al., "Re-evaluation and functional classification of non-synonymous single nucleotide polymorphisms of the human ATP-binding cassette transporter ABCG2," Cancer Science, vol. 98, no. 2, pp. 231-239, 2007.

[79] Y. Imai, M. Nakane, K. Kage et al., "C421A polymorphism in the human breast cancer resistance protein gene is associated with low expression of Q141K protein and low-level drug resistance," Molecular Cancer Therapeutics, vol. 1, no. 8, pp. 611-616, 2002.

[80] D. Kobayashi, I. Ieiri, T. Hirota et al., "Functional assessment of ABCG2 (BRCP) gene polymorphisms to protein expression in human placenta," Drug Metabolism and Disposition, vol. 33, no. 1, pp. 94-101, 2005.

[81] S. Mizuarai, N. Aozasa, and H. Kotani, "Single nucleotide polymorphisms result in impaired membrane localization and reduced atpase activity in multidrug transporter ABCG2," International Journal of Cancer, vol. 109, no. 2, pp. 238-246, 2004.

[82] C. P. Zamber, J. K. Lamba, K. Yasuda et al., "Natural allelic variants of breast cancer resistance protein (BCRP) and their relationship to BCRP expression in human intestine," Pharmacogenetics, vol. 13, no. 1, pp. 19-28, 2003.

[83] K. Morisaki, R. W. Robey, C. Özvegy-Laczka et al., "Single nucleotide polymorphisms modify the transporter activity of ABCG2," Cancer Chemotherapy and Pharmacology, vol. 56, no. 2, pp. 161-172, 2005.

[84] C. Kondo, H. Suzuki, M. Itoda et al., "Functional analysis of SNPs variants of BCRP/ABCG2," Pharmaceutical Research, vol. 21, no. 10, pp. 1895-1903, 2004.
[85] J. Li, G. Cusatis, J. Brahmer et al., "Association of variant ABCG2 and the pharmacokinetics of epidermal growth factor receptor tyrosine kinase inhibitors in cancer patients," Cancer Biology and Therapy, vol. 6, no. 3, pp. 432-438, 2007.

[86] A. Sparreboom, H. Gelderblom, S. Marsh et al., "Diflomotecan pharmacokinetics in relation to ABCG2 421C>A genotype," Clinical Pharmacology and Therapeutics, vol. 76, no. 1, pp. 38-44, 2004.

[87] A. Sparreboom, W. J. Loos, H. Burger et al., "Effect of ABCG2 genotype on the oral bioavailability of topotecan," Cancer Biology \& Therapy, vol. 4, no. 6, pp. 650-658, 2005.

[88] G. Cusatis, V. Gregorc, J. Li et al., "Pharmacogenetics of ABCG2 and adverse reactions to gefitinib," Journal of the National Cancer Institute, vol. 98, no. 23, pp. 1739-1742, 2006.

[89] T. Furukawa, K. Wakabayashi, A. Tamura et al., "Major SNP (Q141K) variant of human ABC transporter ABCG2 undergoes lysosomal and proteasomal degradations," Pharmaceutical Research, vol. 26, no. 2, pp. 469-479, 2009.

[90] T. Ishikawa, A. Sakurai, Y. Kanamori, et al., "High-speed screening of human ATP-binding cassette transporter function and genetic polymorphisms: new strategies in pharmacogenomics," Methods in Enzymology, vol. 400, pp. 485-510, 2005.

[91] M. E. M. Noble, J. A. Endicott, and L. N. Johnson, "Protein kinase inhibitors: insights into drug design from structure," Science, vol. 303, no. 5665, pp. 1800-1805, 2004.

[92] J. Dancey and E. A. Sausville, "Issues and progress with protein kinase inhibitors for cancer treatment," Nature Reviews Drug Discovery, vol. 2, no. 4, pp. 296-313, 2003.

[93] P. Nurse, "Nobel Lecture. Cyclin dependent kinases and cell cycle control," ChemBioChem, vol. 22, no. 5-6, pp. 487-499, 2002.

[94] P. L. Porter, K. E. Malone, P. J. Heagerty et al., "Expression of cell-cycle regulators p27(Kip1) and cyclin E, alone and in combination, correlate with survival in young breast cancer patients," Nature Medicine, vol. 3, no. 2, pp. 222-225, 1997.

[95] J. Tsihlias, L. Kapusta, and J. Slingerland, "The prognostic significance of altered cyclin-dependent kinase inhibitors in human cancer," Annual Review of Medicine, vol. 50, pp. 401423, 1999.

[96] H. Saito, H. Hirano, H. Nakagawa et al., "A new strategy of high-speed screening and quantitative structure-activity relationship analysis to evaluate human ATP-binding cassette transporter ABCG2-drug interactions," Journal of Pharmacology and Experimental Therapeutics, vol. 317, no. 3, pp. 11141124, 2006.

[97] W. Liu, M. R. Baer, M. J. Bowman et al., "The tyrosine kinase inhibitor imatinib mesylate enhances the efficacy of photodynamic therapy by inhibiting ABCG2," Clinical Cancer Research, vol. 13, no. 8, pp. 2463-2470, 2007.

[98] N. Gupta, P. M. Martin, S. Miyauchi et al., "Down-regulation of BCRP/ABCG2 in colorectal and cervical cancer," Biochemical and Biophysical Research Communications, vol. 343, no. 2, pp. 571-577, 2006. 

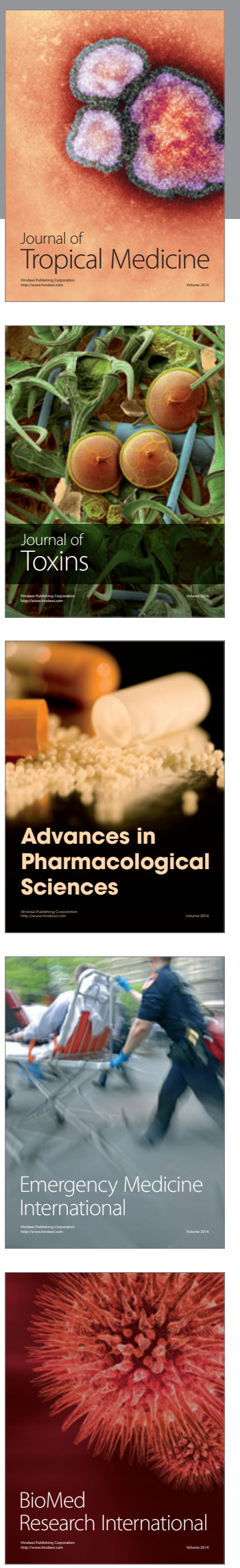
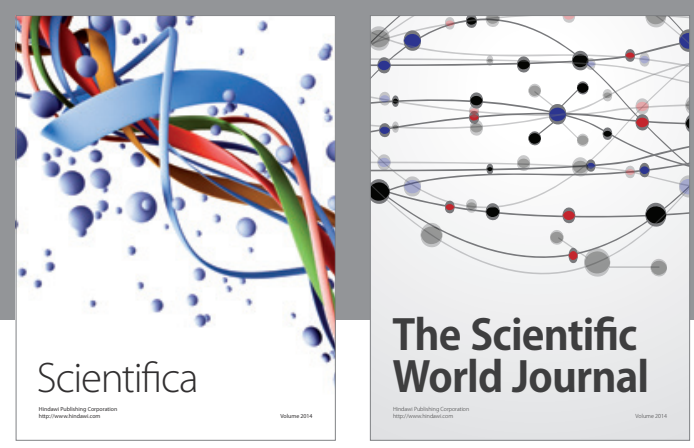

The Scientific World Journal
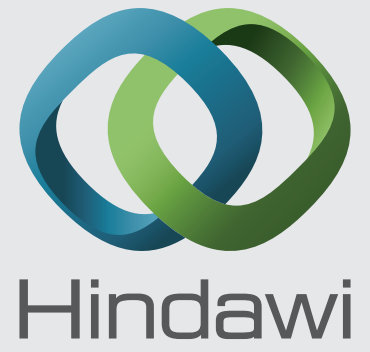

Submit your manuscripts at

http://www.hindawi.com
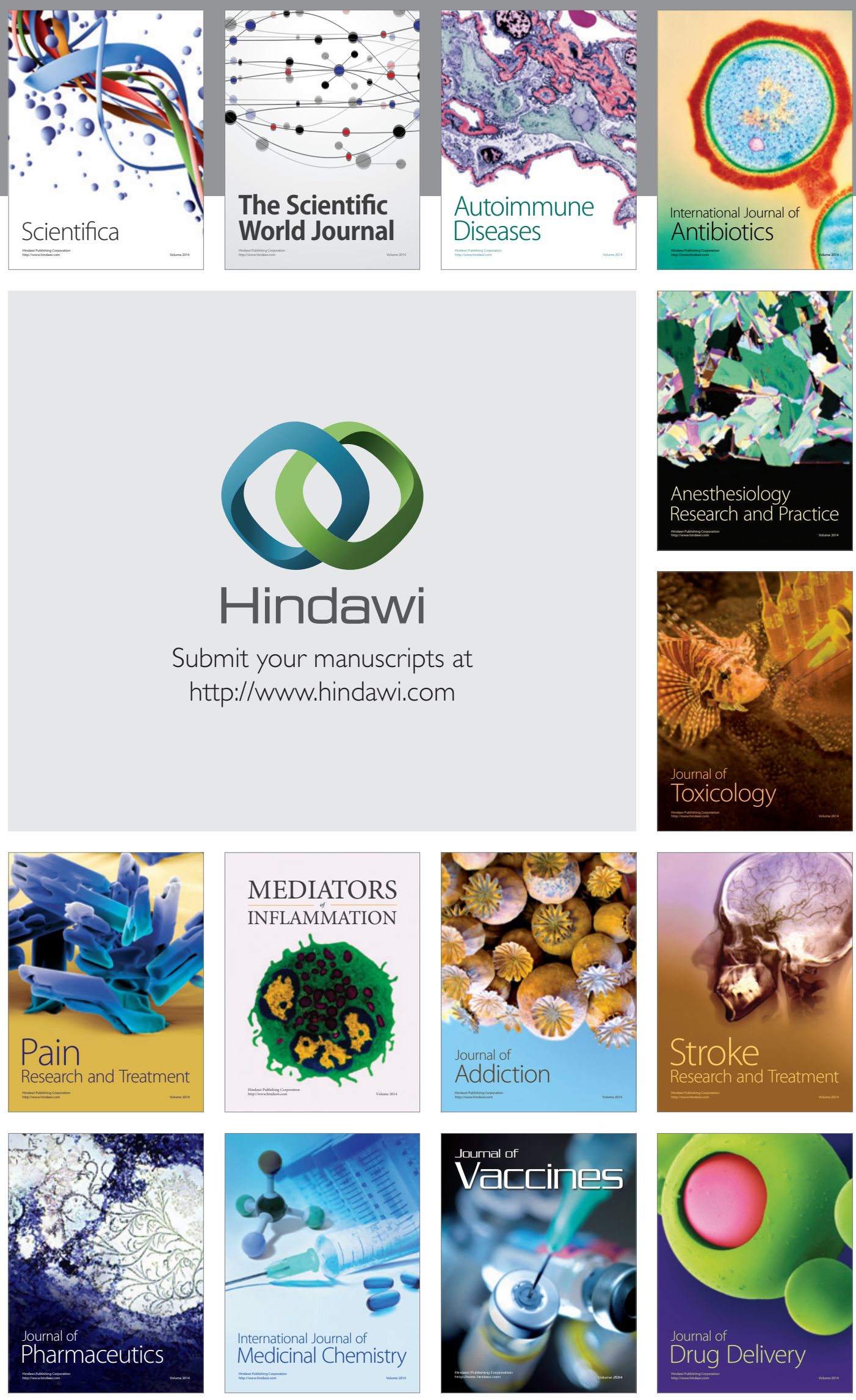